\title{
Build-Up of Burnup Waves in Neutron Absorbing and Diffusive Media
}

\author{
Anoop KV, Kiran Baraik, Om Pal Singh \\ Paper dedicated to memory of Vera Nikolić-Stanojević, \\ Pofessor Emeritus at the State University of Novi Pazar
}

\begin{abstract}
A study has been carried out on the build-up of burn up wave in neutron absorbing and diffusive media. New parameters, i.e., transient length and transient time elapsing in establishing the steady burn up wave, have been introduced. The wave characteristics are expressed in terms of these new parameters and Full Width at Half Maxima (FWHM) and Full Width at 1 $\%$ of Maximum (FW1M). These characterization parameters would be useful in understanding the neutron burn up wave development and their approach to the equilibrium (steady) state.
\end{abstract}

Keywords: Burn up Wave, Neutron Absorber, Diffusive Media, Burnable Poison, Transient Length, Transient Time, Full Width at Half Maximum

\section{Introduction}

In nuclear reactors, neutron absorbing materials are used to control the reactivity. The excess reactivity is compensated by movable control rods consisting of neutron absorbing materials. Alternative method of controlling reactivity is by Burnable Poison (BP) mixed homogeneously with fuel and aiming to achieve reactivity-to-time profile as flat as possible. However, in this approach, to obtain the flattening of reactivity-to-time curve and neutron economy, certain ratio is to be maintained between neutron absorber (poison) cross-section and fuel cross-sections which is difficult to achieve. Another method is to use heterogeneous burnable poison in the form of rods or different regions separate from fuel. In this case, if the dimensions of absorbing region is greater than neutron absorption mean free path in the medium, then the neutron absorption is initially confined only to the outer part of the absorbing region but later it propagates to the inside region. Thus, the burn up of neutron absorber can last for a longer period. Besides this, the burn up of absorber takes place automatically by development of burn up solitary wave in the medium. Some of

Manuscript received April 21, 2014; accepted September 30, 2014.

Anoop KV, Kiran Baraik and Om Pal Singh are with the Indian Institute of Technology Kanpur, Kanpur208 016, India. 
the important references on the control of reactivity and development of solitary burn up wave and soliton theory are such as Duderstadt and Hamilton [1], Rahn et al. [2], Lamb [3], Lomdahl[4] and Lomdahl, et. al., [5], Knief [6], Yashimita [7], Seifritz[8], Van Dam $[9,10,11]$.

One of the initial studies on the propagation of burn up wave of pure absorbing material under neutron irradiation were reported by Seifritz (1995). He analyzed the problem analytically and concluded that neutron reaction rate in the pure absorbing material propagates like a solitary wave. In solitary wave, the spatial shapes of neutron flux and atomic densities of absorbing material do not change during burn up. The burn up wave velocity is related with the ratio of initial neutron flux to neutron absorber density in the medium. The wave gets developed due to non-linearity of equations describing the flux and the atomic densities profile in space and time in the medium. These studies were extended further by Van Dam $[9,10,11]$ to diffusive medium and long term control of excess reactivity by burnable poison in reflectors and burnable particles. Some of the very valuable outcomes of Van Dam studies $[9,10,11]$ include, i) burn up waves in diffusive medium can be applied by locating neutron absorber in reflector region (the core and reflector should be neutronically coupled) like in high temperature gas cooled reactors, ii) long term control on excess reactivity by spherical/cylindrical particles that provide better design flexibility in getting appropriate balance between reactivity loss due to burn up of fuel and reactivity gain by burn up of burnable poison and iii) width of reaction zone is three times the absorption mean free path.

In this work, the results of a study are reported on the characterization of the development and establishment of solitary burn up waves in absorber and diffusive media. To gain insight in the development and establishment of solitons, first the study is carried out for a pure absorbing material in the form of a slab and with a constant neutron flux at one end of the slab. The focus has been on the understanding and then characterization of transient behavior before the soliton like wave gets established. Then, the study is extended to the solitary burn-up wave's development and establishment in a diffusive medium with neutron poison and characterization of solitons. Again a finite slab is considered for the study. Graphite has been taken as a diffusive medium. Boron carbide and Gadolinium are taken as neutron absorbers.

\section{Absorbing medium}

\subsection{Mathematical Model}

The mathematical model used for the study has been adopted from the paper by Seifritz[8] and H. Van Dam [9]. One dimensional semi-infinite slab system is considered for simplicity and to gain better insight in the transient and steady state soliton development in the burn up of pure neutron absorber material under neutron irradiation. A multi energy group and multi-dimensional equation is necessary to obtain realistic quantitative results. The simple model adopted is good enough to demonstrate the utility of the new parameters used in characterizing the solitary wave development in a pure neutron absorbing medium.

A neutron beam with flux, $\Phi(\mathrm{x}, \mathrm{t})$ is incident perpendicular to the boundary of the slab 
at $\mathrm{x}=0$. The space and time dependent neutron flux, $\Phi(\mathrm{x}, \mathrm{t})$ and the neutron absorber nuclide density $N(x, t)$ can be expressed by following the Partial Differential Equations [9]:

$$
\begin{aligned}
& \frac{\partial \Phi(\mathrm{x}, \mathrm{t})}{\partial \mathrm{x}}=-\sigma \mathrm{N}(\mathrm{x}, \mathrm{t}) \Phi(\mathrm{x}, \mathrm{t}) \\
& \frac{\partial \Phi(\mathrm{x}, \mathrm{t})}{\partial \mathrm{t}}=-\sigma \mathrm{N}(\mathrm{x}, \mathrm{t}) \Phi(\mathrm{x}, \mathrm{t})
\end{aligned}
$$

where

$$
\begin{aligned}
& \Phi=\text { Neutron flux } \\
& \mathrm{N}=\text { Neutron absorber nuclide density } \\
& \sigma=\text { Absorber's microscopic absorption cross-section }
\end{aligned}
$$

Equations (1) and (2) are solved numerically with finite difference technique using initial and boundary conditions as given by Seifritz W., (1995). That is,

$$
\begin{array}{lll}
\Phi(0,0)=\Phi_{\mathrm{o}} & \Phi(0, \mathrm{t})=\Phi_{\mathrm{o}}, & \Phi(\mathrm{x}, 0)=\Phi_{\mathrm{o}} \exp \Phi\left(-\sigma \mathrm{N}_{\mathrm{o}} \mathrm{x}\right) \\
\mathrm{N}(0,0)=\mathrm{N}_{\mathrm{o}} & \mathrm{N}(\mathrm{x}, 0)=\mathrm{N}_{\mathrm{o}} & \mathrm{N}(0, \mathrm{t})=\mathrm{N}_{\mathrm{o}} \exp \Phi\left(-\sigma \Phi_{\mathrm{o}} \mathrm{t}\right)
\end{array}
$$

The subscript $O$ represents the initial value of the parameters. The Reaction Rates, $\mathrm{R}(\mathrm{x}, \mathrm{t})$ are calculated using the expression,

$$
\mathrm{R}(\mathrm{x}, \mathrm{t})=\sigma \phi(\mathrm{x}, \mathrm{t}) \mathrm{N}(\mathrm{x}, \mathrm{t})
$$

\subsection{Results and Discussions}

\subsubsection{Data}

Natural boron carbide is considered as the neutron absorbing medium. Data are taken from [9]. A neutron beam with flux, $10^{14} \mathrm{~cm}^{-2} \cdot \mathrm{s}^{-1}$ is assumed to be incident perpendicularly on the boundary of the natural boron carbide slab at $\mathrm{x}=0$. Natural boron carbide density is taken as $2.52{\mathrm{~g} . \mathrm{cm}^{-3}}^{-3}$ and thermal cross-section of $\mathrm{B}^{10}$ is taken as 3847 barns. Only $\mathrm{B}^{10}$ is taken in the analysis because $\mathrm{B}^{11}$ is transparent to neutrons. The absorber $\left(\mathrm{B}^{10}\right)$ nuclide density is $2.2 \times 10^{22} \mathrm{~cm}^{-3}$.

\subsubsection{Transient and Steady State Phase Characteristics}

The results of the calculations of neutron flux and absorber nuclide density as a function of space and time are presented in Figs. 1 and 2. Figure 3 shows the reaction rate density distribution in space and time. Each curve is plotted at an interval of 10 days and covers a period of 250 days. In the figures, one can see gradual development of asymptotic (steady 


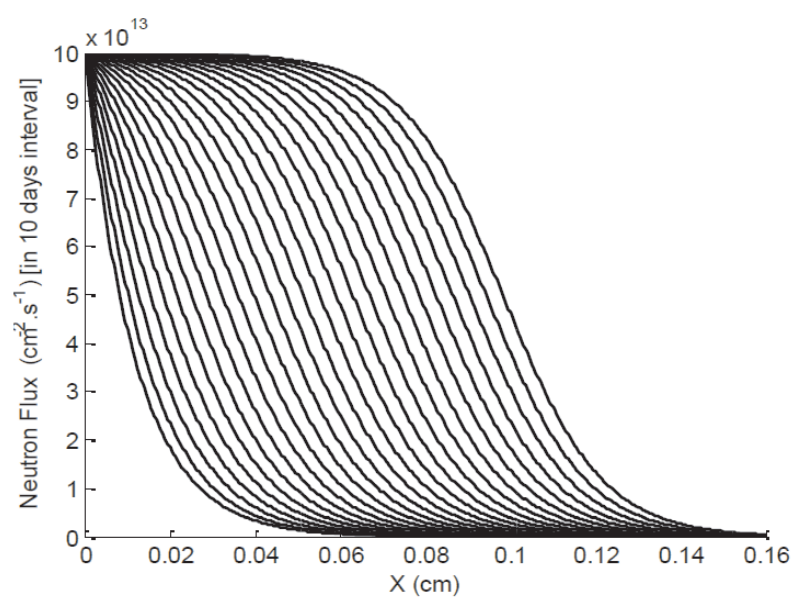

Fig. 1. Neutron flux vs. space (X)

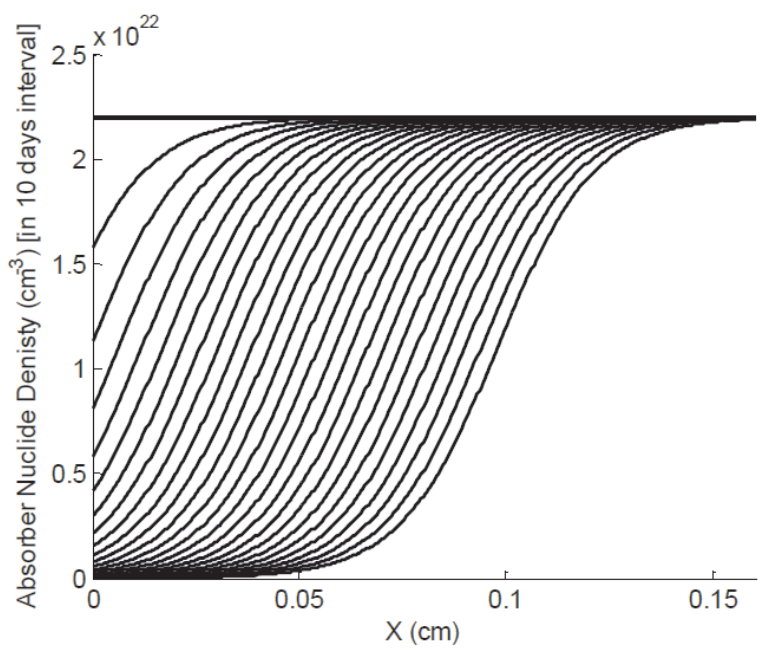

Fig. 2. Absorber nuclide density Vs space (X)

state) burn up wave through transient phase. The shape of neutron flux and nuclide density in the transient state changes and finally attains steady state shape. These figures are characterized by the following parameters.

\section{Transient Phase}

i) Transient Time (TT): This is defined as the time elapsed in attaining the asymptotic (steady) state of the burn up wave. This can be considered as the duration of transient phase. To quantify the TT, two criteria are employed: a) the time at which the wave attains $95 \%$ (represented by TT 95) of its steady state and b) the time at which it attains $99 \%$ (represented by TT 99) of its steady state value. Often, in such types 


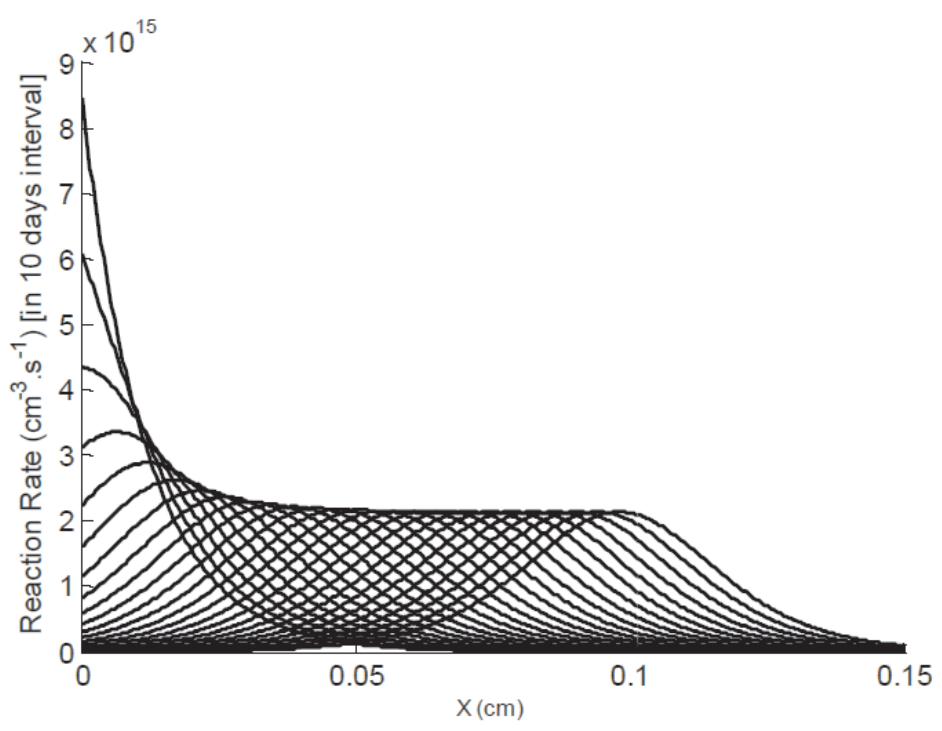

Fig. 3. Reaction rate vs. space (X)

of problems, the transient phase changes rapidly in the beginning and quite slowly when the steady state is approaching. In view of this, we thought it prudent to have the above two criteria to define the transient time.

ii) Transient Length (TL): This is the distance covered by the wave to achieve steady state shape. In analogy to the transient time, transient lengths are also defined as TL95 and TL99; that is, the length at which the wave attains $95 \%$ (represented by TL 95) of its steady state and the length at which it attains $99 \%$ (represented by TL 99) of its steady state value.

\section{Steady State Phase:}

i) Wave Velocity: This is the velocity of the burn up wave propagation. Theoretically, it is calculated as the ratio of initial flux to the initial nuclide density.

ii) Reaction Rate Zone Width:

- Full Width at Half Maximum (FWHM): This is the width of reaction rate curve between the half values of the maximum reaction rate.

- Full Width at $1 \%$ of the Maximum (FW1M): This is the width of reaction rate curve between the $1 \%$ values of the maximum reaction rate.

The wave velocity and FWHM are always used in characterization of waves. However, FW1M has been introduced to get the quantitative idea of the width of reaction rate curve at the two ends of curve. 
The above parameters are general and can also be applied for characterization of solitary burn up wave development in diffusive and multiplying media. In fact, for designing any system based on the solitary wave development, the above burn up wave parameters can serve as design parameters for optimal design of the system.

In the present study, the above parameters are calculated by full numerical simulation of transient phase as well as steady state phase. The salient features of the characteristics of absorber burn up wave are as follows.

1. The transient time is calculated from the solitary wave developed in neutron flux (Fig. 1), absorber nuclide density (Fig. 2) and reaction rate (Fig. 3). However, we found the Fig. 3 more convenient to extract the transient times, i.e., TT95 and TT99. The values of TT95 and TT99 are found to be 90 days and 138 days respectively. These values turn out to be 3 and 4.6 times of the characteristic time, $t\left(=\frac{1}{s \Phi_{0}}=30\right.$ days) of the system. It is observed that $\frac{N(t)}{N_{o}}\left(=\exp \left(-\frac{t}{t}\right)\right)$ reduces by $95 \%$ and $99 \%$ after $\mathrm{t}=3 \tau$ and $\mathrm{t}=4.6 \tau$ respectively. This is in conformity with the analytical results of Seifritz (1995).

2. The transient length (TL95 and TL99) is calculated by finding the length corresponding to the transient time of 90 days and 138 days respectively. It is found that TL95 $=0.035 \mathrm{~cm}$ and TL99 $=0.055 \mathrm{~cm}$. The TL95 and TL99 are connected with the mean free path, $\Phi\left(=\frac{1}{s \mathrm{~N}_{\mathrm{o}}}=0.012 \mathrm{~cm}\right)$ of neutrons in the medium. These are about 3 times of $\lambda$ for TL95 and 4.6 times of $\lambda$ for TL99. These values are reduction in the values of $\frac{\Phi(\mathrm{t})}{\Phi_{\mathrm{o}}}\left(=\exp \left(-\frac{\mathrm{x}}{\lambda}\right)\right)$, by $95 \%$ and $99 \%$ after $\mathrm{x}=3 \lambda$ and $\mathrm{x}=4.6 \lambda$ respectively.

3. From the simulation, the velocity of the burnup wave is found to be equal to $0.14 \mathrm{~cm}$ per year which is equal to the theoretically expected value obtained from initial flux and initial neutron absorber nuclide density ratio, $\frac{\Phi_{\mathrm{o}}}{\mathrm{N}_{\mathrm{o}}}$.

4. The width of the space (reaction rate zone) over which the steady state burn up wave is progressing is calculated from Fig. 3. The FWHM is found to be $0.04 \mathrm{~cm}$ which is 3.5 times the neutron mean free path in the medium. The FW1M $0.12 \mathrm{~cm}$ which is about 10 times the neutron mean free path of the medium and three times the FWHM.

It can be seen that the transient phase characteristics (TT95 and TT99) are connected with the characteristic time, $t=\frac{1}{s \Phi_{0}}$ of the medium and TL95 and TL99 are connected with mean free path of the medium, $\Phi=\frac{1}{s \mathrm{~N}_{\mathrm{o}}}$. The width of the reaction rate curve is also connected with the neutron mean free path of the medium. One can calculate these characteristics for any combination of initial flux, initial absorber nuclide density and enrichment of the neutron absorber. The designers of the system would decide, based on the values of these parameter, on the practical utility of the solitary burn up wave development.

\subsubsection{Burn up in Different Media}

The characteristics of nuclear solitary burn up wave have been studied for different neutron absorbers (poisons) like cadmium, europium and hafnium. The cross-section data for the 
absorbers are taken from Van Dam (2000b). The properties of different absorbers used are given in Table 1. For each neutron absorber, the mean free path and characteristic time will be different depending on their absorption cross-section and the abundance. The values of these parameters are given in Table 2. The values of TTs and TLs are expressed in absolute values as well as in terms of characteristic time and mean free path.

Table 1. Properties of Different Neutron Absorbers (Poisons)

\begin{tabular}{ccccc}
\hline Absorbers & $\begin{array}{c}\text { Natural Abundance } \\
(\%)\end{array}$ & $\begin{array}{c}\text { Cross }- \\
\text { Sections } \\
\text { (barn) }\end{array}$ & $\begin{array}{c}\text { Number density } \\
\text { Neutrons } / \mathrm{cm}^{3}\end{array}$ & $\begin{array}{c}\text { Characteristic Time, } \tau \\
\text { (days) }\end{array}$ \\
\hline B-10 & 20 & 3847 & $4.4 \times 10^{22}$ & 30.1 \\
Cd-113 & 12.22 & 20767 & $5.6636 \times 10^{21}$ & 5.57 \\
Sm-149 & 13.8 & 41000 & $4.1570 \times 10^{21}$ & 2.82 \\
Eu-151 & 47.8 & 9180 & $9.9728 \times 10^{21}$ & 12.61 \\
Hf-177 & 13.31 & 377 & $8.3566 \times 10^{21}$ & 30.7 \\
\hline
\end{tabular}

Table 2. Characteristic Properties of Solitary Neutron Burn up Wave in Different Absorbers

\begin{tabular}{|c|c|c|c|c|c|c|c|}
\hline \multirow{2}{*}{ Absorbers } & \multirow{2}{*}{$\begin{array}{l}\text { Velocity } \\
\text { of } \\
\text { burnup } \\
\text { wave } \\
\mathrm{cm} / \text { year }\end{array}$} & \multirow{2}{*}{$\begin{array}{l}\text { Mean } \\
\text { free } \\
\text { path } \\
(\lambda) \\
(\mathrm{cm})\end{array}$} & \multicolumn{2}{|c|}{ Transient Length (cm) } & \multicolumn{2}{|c|}{ Transient Time (days) } & \multirow{2}{*}{$\begin{array}{c}\text { FWHM } \\
\text { of } \\
\text { Reaction } \\
\text { Rate } \\
\text { (cm) }\end{array}$} \\
\hline & & & $\begin{array}{c}\text { Absolute } \\
\text { Value }\end{array}$ & (in $\lambda$ ) & $\begin{array}{c}\text { Absolute } \\
\text { Value }\end{array}$ & (in $\tau$ ) & \\
\hline B-10 & 0.72 & 0.590 & 0.28 & $4.6 \lambda^{\mathrm{B}-10}$ & 140 & $4.6 \tau^{\mathrm{B}-10}$ & 0.206 \\
\hline Cd-113 & 0.56 & 0.009 & 0.04 & $4.6 \lambda^{\mathrm{Cd}-113}$ & 25 & $4.6 \tau^{\mathrm{Cd}-133}$ & 0.029 \\
\hline Sm-149 & 0.76 & 0.006 & 0.03 & $4.6 \lambda^{\mathrm{Sm}-149}$ & 13 & $4.6 \tau^{\mathrm{Sm}-149}$ & 0.020 \\
\hline Eu-151 & 0.32 & 0.011 & 0.05 & $4.6 \lambda^{\mathrm{Eu}-151}$ & 57 & $4.6 \tau^{\mathrm{Eu}-151}$ & 0.038 \\
\hline Hf-177 & 0.38 & 0.317 & 1.45 & $4.6 \lambda^{\mathrm{Hf}-177}$ & 1400 & $4.6 \tau^{\mathrm{Hf}-177}$ & 1.110 \\
\hline
\end{tabular}

\subsubsection{TT and TL dependence on applied Criteria}

The dependence of Transient Length and Transient Time on the Criteria applied on establishment of asymptotic (steady state) burn up wave was studied. The results are given in Table 3. From the table, it is observed that the transient time and transient length are decreasing with percentage criteria employed. The relationship of TT with $t$ goes down from Transient time $=4.6 \tau$ for achieving $99 \%$ of asymptotic value to Transient Time $=1.3 \tau$ for achieving $70 \%$ of asymptotic value. Similarly, the relationship of TL with $\lambda$ goes down from Transient length $=4.61$ for achieving $99 \%$ of asymptotic value to Transient length $=$ 1.31 for achieving $70 \%$ of asymptotic value. 
Table 3. Transient Length and Transient Time dependence on the Criteria for development of Asymptotic Wave

\begin{tabular}{|c|c|c|c|c|}
\hline \multirow{2}{*}{$\begin{array}{l}\text { Approximate } \\
\text { Criteria (\% of } \\
\text { asymptotic } \\
\text { value) }\end{array}$} & \multicolumn{2}{|c|}{ Transient Length } & \multicolumn{2}{|c|}{ Transient Time } \\
\hline & Absolute (mm) & in $(\lambda)$ & Absolute (days) & in $(\tau)$ \\
\hline 99 & 0.55 & $4.6 \lambda$ & 140 & $4.6 \tau$ \\
\hline 95 & 0.35 & $3.0 \lambda$ & 90 & $3.0 \tau$ \\
\hline 90 & 0.26 & $2.2 \lambda$ & 70 & $2.3 \tau$ \\
\hline 80 & 0.16 & $1.5 \lambda$ & 47 & $1.6 \tau$ \\
\hline 70 & 0.10 & $1.3 \lambda$ & 35 & $1.3 \tau$ \\
\hline
\end{tabular}

\section{Diffusive Medium}

In a pure absorber case, scattering of the neutrons by nuclides is negligible. But if we need to increase the velocity of the burnup wave absorber density should be diluted by adding neutron moderating material. The neutron flux distribution and burnup characteristics of a diffusive medium with absorbers can be calculated using diffusion equation and burnup equation simultaneously. Van Dam [7] studied the problem by considering the following equations.

$$
\mathrm{D} \frac{\partial^{2} \Phi(\mathrm{x}, \mathrm{t})}{\partial \mathrm{x}^{2}}-\sigma \mathrm{N}(\mathrm{x}, \mathrm{t})=\frac{1}{\mathrm{v}} \frac{\partial \Phi(\mathrm{x}, \mathrm{t})}{\partial \mathrm{t}}
$$

where $\mathrm{D}=$ Diffusion coefficient, $\mathrm{v}=$ Neutron Velocity and other symbols have same meaning as defined earlier. It is assumed that the scattering material will not absorb any neutrons. For the sake of simplicity, slab geometry is considered.

The time derivative term in the above equation can be neglected since variation in flux with time is very slow and neutron velocity is large (2200 m/s for thermal neutrons). So the Eq. (5) becomes,

$$
\mathrm{D} \frac{\partial^{2} \Phi(\mathrm{x}, \mathrm{t})}{\partial \mathrm{x}^{2}}-\sigma \mathrm{N}(\mathrm{x}, \mathrm{t}) \Phi(\mathrm{x}, \mathrm{t})=0
$$

The burnup equation for poison density is,

$$
\frac{\partial \mathrm{N}(\mathrm{x}, \mathrm{t})}{\partial \mathrm{t}}+\sigma \mathrm{N}(\mathrm{x}, \mathrm{t}) \Phi(\mathrm{x}, \mathrm{t})=0
$$

The Initial and boundary conditions for the problem are:

$$
\mathrm{J}(0, \mathrm{t})=\mathrm{J}_{0} \quad \mathrm{~N}(\mathrm{x}, 0)=\mathrm{N}_{0} \quad \Phi(100, \mathrm{t})=\Phi_{0}
$$

where $\mathbf{J}=$ Neutron current density. Flux is assumed to vanish at boundary. Graphical representation of a slab with $100 \mathrm{~cm}$ length is given below:

The Eq. (6) and Eq. (7) are solved by numerical methods. Runge-Kutta and finite difference methods are used. Using initial conditions, the neutron flux can be found analytically for 


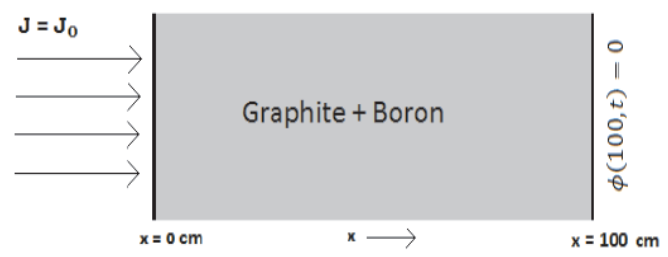

Fig. 4. Slab geometry

all $\mathrm{x}$. This is used to obtain absorber density, with this, we can setup a system of equations which can be solved by Gaussian elimination method to obtain neutron flux for all ' $x$ ' at a particular time. Once flux and absorber density are known at a time, fourth order RungeKutta method is applied to burn up equation to get the absorber density at next time step for all ' $x$ '.

The transient characteristics of burn up wave are studied in detail. To know the variation of characteristics in different media simulation is done with different Diffusion Coefficients. Input data used are from [9]:

1. Net input neutron current $\left(\mathrm{J}_{0}\right)=10^{14} \mathrm{~cm}^{-2} \mathrm{~s}^{-1}$

2. Diffusion coefficient $(D)=1 \mathrm{~cm}$

3. Boron-10 density $\left(\mathrm{N}_{0}\right)=1.04 \times 10^{19} \mathrm{~cm}^{-3}$

4. B-10 absorption cross section $(\sigma)=3847$ barns

5. Slab length $=100 \mathrm{~cm}$

From the above data we get diffusion length, $\mathrm{L}=5 \mathrm{~cm}$. Diffusion length is calculated using the equation,

$$
\mathrm{L}=\sqrt{\frac{\mathrm{D}}{\mathrm{N}_{\mathrm{o}} \sigma}}
$$

Theoretical velocity of the reaction zone is given by $\frac{\mathrm{J}_{\mathrm{o}}}{\mathrm{N}_{\mathrm{o}}}=303 \mathrm{~cm} /$ year. The characteristic time is 6 days. The profile of neutron flux, absorber density and reaction rate as a function of space and time are shown in Figs. 5, $6 \& 7$ respectively. The change from transient state to asymptotic state of reaction rate is clearly demonstrated. Following observations are made from this simulation.

1. The velocity of the burn up wave is found to be $303 \mathrm{~cm} / \mathrm{year}$ which is as expected.

2. The burn up wave gets established to $99.9 \%$ of its asymptotic value after 21 days. This is 3.4 times of characteristic time. 
3. Corresponding to a transient time of 21 days, transient length is $15 \mathrm{~cm}$. It is equal to three times the diffusion length.

4. The width of the slab over which reaction is taking place is quantified in terms of FWHM. FWHM in this case is found to be $14 \mathrm{~cm}$.

In Fig.8, reaction rate distribution is plotted for $60^{\text {th }}$ day. It shows that the distribution is asymmetric. The shape of the reaction rate distribution is similar to that of spatial derivative of absorber distribution as shown in Fig. 9.

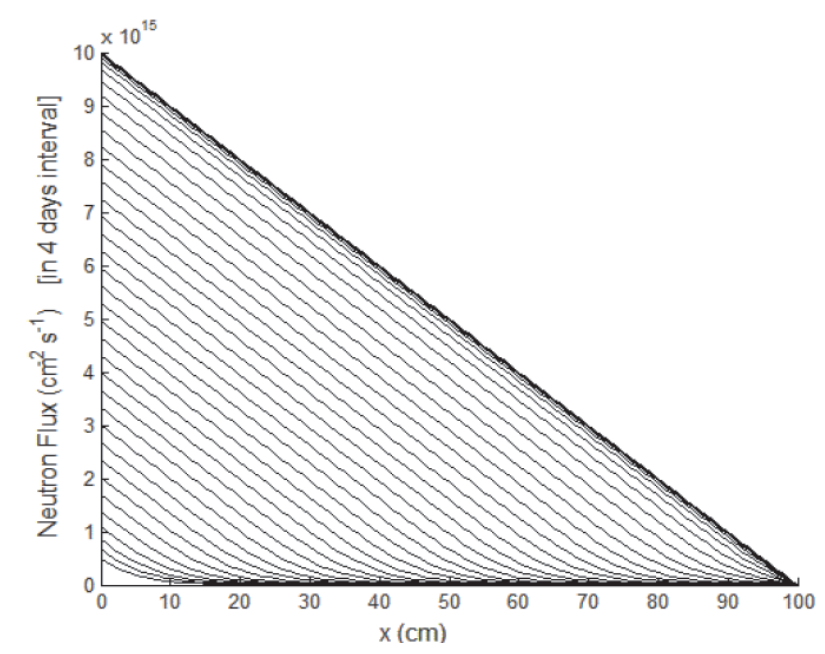

Fig. 5. Neutron flux vs. space (X)

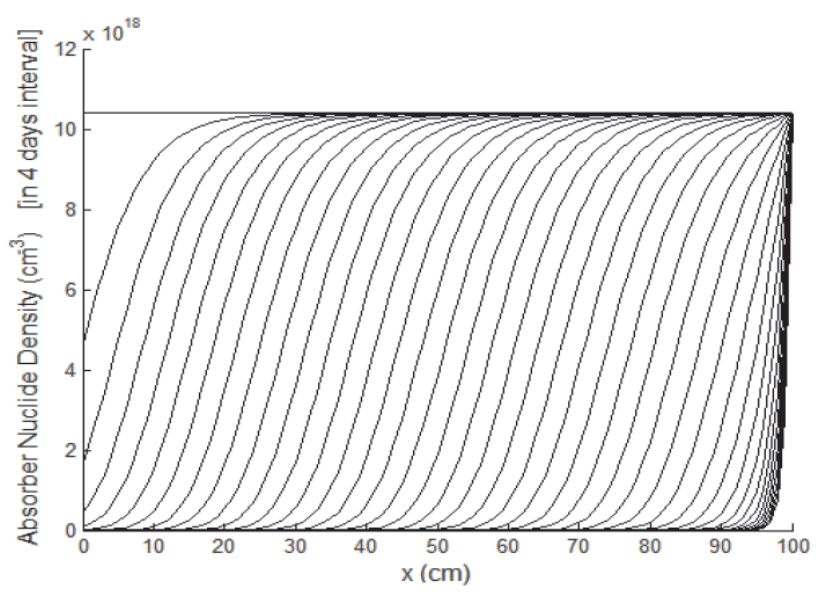

Fig. 6. Absorber nuclide density vs. space (X)

A parametric study has been made with respect to Diffusion Coefficient. Simulations were performed to study the effect of different medium on burn up wave propagation. The 


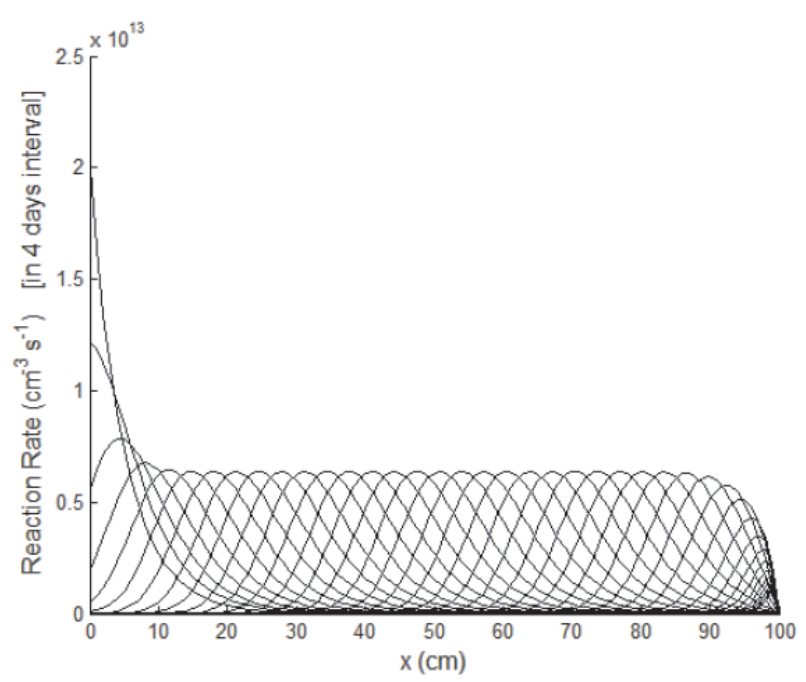

Fig. 7. Reaction rate vs. space at 2 days intervals

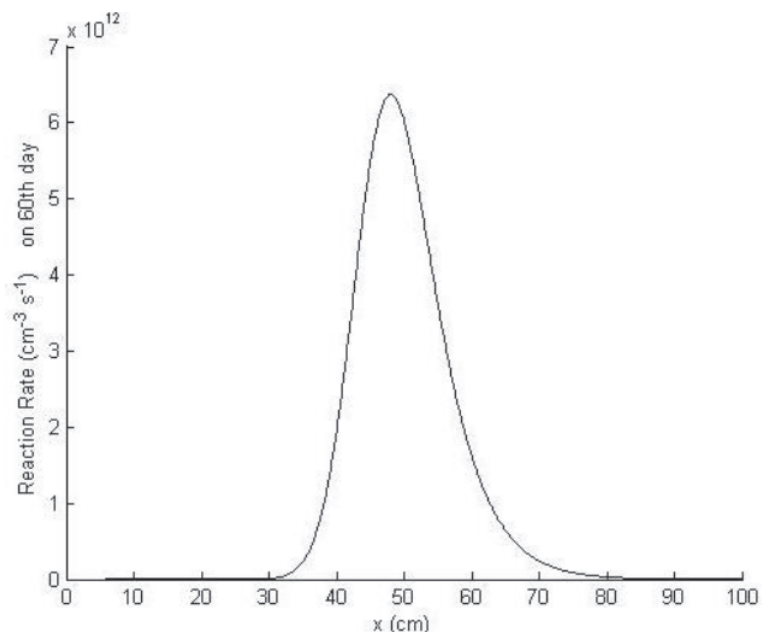

Fig. 8. Reaction rate distribution on $60^{\text {th }}$ day

results are given in Table 4. Following conclusions can be made from the results.

1. The calculated burn up wave velocity as expected is independent of diffusion coefficient

2. When diffusion coefficient increases (i.e., diffusion length increases), transient length also increases. It is also observed that ratio between transient length and diffusion length remains constant and is equal to 3.0

3. Transient time increases with increasing diffusion coefficient. Here transient time to characteristic time ratio remains constant and is equal to 3.4 


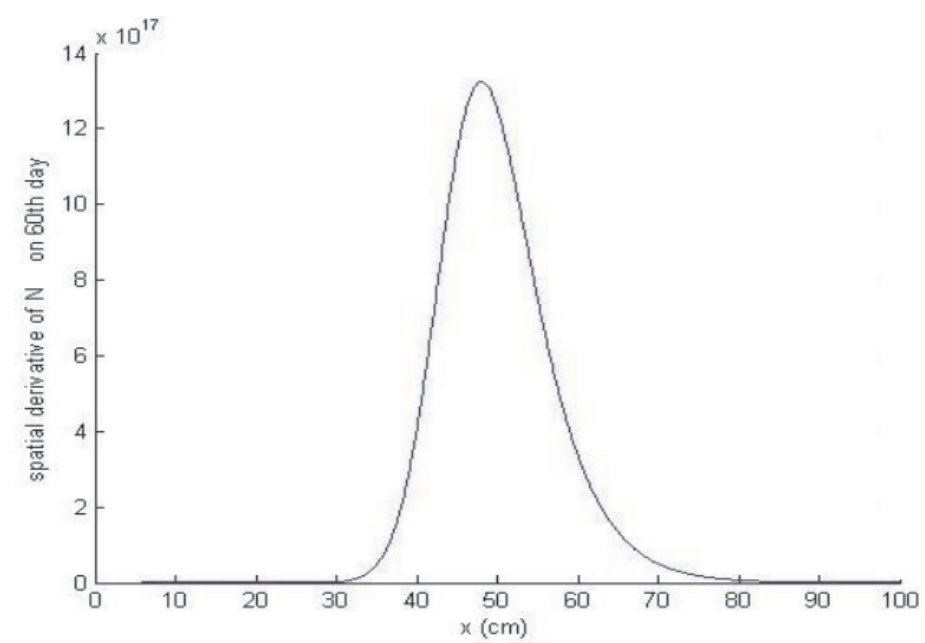

Fig. 9. Spatial derivative of absorber density on $60^{\text {th }}$ day

Table 4. Simulation results for different diffusion coefficients

\begin{tabular}{|c|c|c|c|c|c|c|c|}
\hline \multirow[t]{2}{*}{ Parameters } & \multicolumn{7}{|c|}{$\begin{array}{l}\text { With scattering medium (Transition time : } 99.9 \% \text { of Reaction } \\
\text { Rate Peak) }\end{array}$} \\
\hline & $\mathrm{D}=0.001$ & $\mathrm{D}=0.1$ & $\mathrm{D}=1.0$ & $\mathrm{D}=5$ & $\mathrm{D}=10$ & $\begin{array}{l}\mathrm{D}= \\
25\end{array}$ & $\begin{array}{l}\mathrm{D}= \\
100\end{array}$ \\
\hline Velocity (cm/year) & 302 & 302 & 303 & 302 & 302 & 302 & 302 \\
\hline $\begin{array}{l}\text { Transient Length } \\
(\mathrm{cm})\end{array}$ & 0.48 & 4.7 & 15.1 & 33.5 & 48 & 75.5 & 151 \\
\hline $\begin{array}{l}\text { Diffusion Length } \\
\text { (cm) } \\
\text { Transient Length / }\end{array}$ & 0.16 & 1.58 & 5 & 11.18 & 15.81 & 25 & 49.99 \\
\hline $\begin{array}{l}\text { Diffusion Length } \\
\text { Transient Time }\end{array}$ & 3.00 & 2.97 & 3.02 & 3.00 & 3.04 & 3.02 & 3.02 \\
\hline $\begin{array}{l}\text { (days) } \\
\text { Characteristic time }\end{array}$ & 0.66 & 6.47 & 20.72 & 46.05 & 65.3 & 103.55 & 207.15 \\
\hline $\begin{array}{l}\text { (days) } \\
\text { Transient Time / }\end{array}$ & 0.19 & 1.9 & 6.02 & 13.46 & 19.03 & 30.09 & 60.18 \\
\hline Characteristic Time & 3.47 & 3.41 & 3.44 & 3.42 & 3.43 & 3.44 & 3.44 \\
\hline $\begin{array}{l}\text { FWHM }(\mathrm{cm}) \\
\text { FWHM / }\end{array}$ & 0.45 & 4.47 & 14.13 & 31.61 & 44.7 & 70.68 & 141.36 \\
\hline Diffusion Length & 2.81 & 2.83 & 2.83 & 2.83 & 2.83 & 2.83 & 2.83 \\
\hline
\end{tabular}

4. The FWHM of the burn up wave also increases with diffusion coefficient. It is 2.8 times the diffusion length.

A set of studies have been done with Gadolinium as absorber. The simulation results and comparison with boron medium are given in Table 5. Initial Gadolinium density is chosen such as in such a way that diffusion length in both media is same. 
Table 5. Gadolinium as absorber

\begin{tabular}{lcc}
\hline \multicolumn{1}{c}{ Parameters } & B-10 & Gd-157 \\
\hline Initial Absorber Density ( & $1.04 \mathrm{E}+19$ & $1.58 \mathrm{E}+17$ \\
${\text { atoms } / \mathrm{cm}^{3} \text { ) }}^{\text {Absorption Cross Section (barns) }}$ & 3847 & 254,000 \\
Velocity simulation (cm/year) & 303 & $55 \mathrm{~cm} /$ day \\
Velocity analytical (cm/year) & 303 & $54 \mathrm{~cm} /$ day \\
Transient Length (cm) & 15.0 & 15.5 \\
Diffusion Length (cm) & 5 & 5 \\
Transient length / Diffusion length & 3.0 & 3.1 \\
Transient Time (days) & 20.7 & 0.3 \\
Characteristic time (days) & 6.0 & 0.1 \\
Transient time / characteristic time & 3.4 & 3.7 \\
FWHM (cm) & 14 & 14 \\
RFWHM/ Diffusion length & 2.8 & 2.8 \\
\hline
\end{tabular}

\section{Conclusions}

Efforts are made to characterize the solitary burn up wave development and propagation in a pure neutron absorbing medium and in a diffusive medium. The neutron absorbing medium considered is boron carbide and diffusive medium is graphite with boron carbide. Numerical simulation approach is followed.

The wave is characterized in terms of velocity of propagation of solitary wave, transient times and length (the time and length that soliton takes and covers to reach equilibrium (asymptotic) shape) involved in establishment of asymptotic solitary burn up wave and width of the reaction zone. The width of the wave spread is expressed in terms of Full Width at Half Maximum (FWHM) of reaction rates and Width at $99 \%$ Area (W99A) under the curves of reaction rates.

The paper provides a new approach and new parameters to qualify the transient part of the soliton and its connection with intrinsic and design parameters of the reactor.

\section{References}

[1] Duderstadt J. J. and Hamilton L. J.,Nuclear Reactor Analysis, Wiley, New York, (1976).

[2] Lamb G.I., Elements of Soliton Theory, Wiley, London, (1980).

[3] Lomdahl, P.S.,What is a Soliton? Los Alamos Science, 10, 27,(1984).

[4] Lomdahl P.S.; Layne, S.P. and Bijio, I. J., Solitons in Biology? Los Alamos Science, 10, 3 , (1984).

[5] Rahn F. J., Adamtiades A. G.,Kenton J.E. and Braun C., A Guide to Nuclear Power Technology, Wiley, New York, (1984).

[6] Knief R. A., Nuclear Engineering, Hemisphere Publication, (1992). 
[7] Yamashita K., Journal of Nuclear Science and Technology, (31, 979-985, (1994).

[8] Seifritz W., Non-linear Burn-up Waves in Opaque Neutron Absorbers, Kerntechnik, 60, 185 188, (1995).

[9] ] Van Dam Hugo,Burnup Waves, Annals of Nuclear Energy, Vol. 25, No. 17, 14091417,(1998).

[10] Van Dam Hugo,Long-Term Control of Excess Reactivity by Burnable Poison in Reflector Regions, Annals of Nuclear Energy, 27, 63-69, (2000a).

[11] Van Dam Hugo,Long-Term Control of Excess Reactivity by Burnable Particles, Annals of Nuclear Energy, 27, 733, (2000b). 